Equally, in the limited format of a request form, omission of key "trigger" words, in an otherwise correctly com-

\title{
Rational requesting or rationing testing?
}

R J Lock

\section{The appropriate use of antineutrophil cytoplasm antibody (ANCA) tests}

$\mathrm{T}$ he UK government has acknowledged that up to $70 \%$ of medical diagnoses now rely on pathology laboratory analyses. ${ }^{1}$ One consequence of such reliance is an ever increasing laboratory workload, usually, in National Health Service laboratories, at a rate in excess of the financial resources to support it. It follows that this demand can only be serviced by increased efficiency.

One area where demand has risen sharply in the past decade is in the field of antineutrophil cytoplasmic antibody (ANCA) testing. Since the association of ANCA with Wegener's granulomatosis (WG) in $1985,{ }^{2}$ an increasing number of autoimmune, drug induced, and infectious disorders have been shown to have an association with ANCA. $^{3-8}$ However, overall, the data suggest that these ANCA do not help to elucidate the diagnosis or prognostic features of these disorders (reviewed in Savige et al, 2000'). In addition, there is increasing evidence to suggest that ANCA may be of help, albeit limited, in categorising inflammatory bowel disease. ${ }^{10}{ }^{11}$ Low sensitivity limits the diagnostic use of ANCA in this area. ${ }^{12} 13$

As indicated in the consensus document, ${ }^{14}$ the principle use of ANCA testing remains as an aid to diagnosis in the necrotising vasculitides (such as WG, microscopic polyangiitis, ChurgStrauss, and pauci-immune crescentic glomerulonephritis). Untreated, these conditions have considerable morbidity and mortality.

In this issue of the Journal of Clinical Pathology, Sinclair et al have reviewed their experience over a six month period of a "gating policy", based on clinical information given to the laboratory at the time of request, which has been in place for 10 years. ${ }^{15}$ This policy directs requesting almost exclusively towards the diagnosis of the necrotising vasculitides. Their data suggest that their strategy significantly reduces ANCA requesting and they contend that this has not resulted in missed or greatly delayed diagnoses. "open door" testing service; that is, unrestricted testing of all samples arriving in the laboratory without regard to clinical background. Our own experience shows that in this scenario a large number of immunofluorescent ANCA are detected outside the context of necrotising vasculitis. ${ }^{16}$ Further retrospective studies have confirmed that open door testing has a low yield. ${ }^{17-19}$ In these circumstances, the positive predictive value (PPV) of the assay for the necrotising vasculitides is very low. Indeed, McLaren et al showed that in the context of neurological disease the PPV was $0 \%$, at an estimated cost of $£ 12000$ over a four year period. ${ }^{19}$ With increasing attention to context and to symptomoldisease. ${ }^{20}$

\section{"The potential cost to the patient and to the service of a missed diagnosis of glomerulonephritis could be extensive, resulting for example in plasma exchange or dialysis"}

Of some concern, the present study relies very heavily on the quality of clinical data included on request forms. This has several implications for the laboratory. First, personal experience suggests that request forms are often inadequately completed, many with no clinical details included at all. These are rejected under the Sinclair rules. Second, who is the gatekeeper? A senior member of staff would need to dedicate a considerable amount of time to this additional task. We receive over 5000 ANCA requests each year. At 30 seconds to review each form this would equate to 42 hours each year, a full working week. Third, electronic requesting is increasingly available. This uses coded comments available to the clinician, including codes such as "vasculitis screen", and effectively bypasses the request form review.

More usually, laboratories offer an ogy the PPV can be greatly improved, being highest in those with renal

pleted form, may lead to unintentional yet unwarranted delay in the diagnostic process. Indeed, Sinclair et al describe one such case in their series. The patient was initially "query mixed connective tissue disease" and only later were the data of "episcleritis, haematuria, and proteinuria" made available. WG, in keeping with many autoimmune disorders, may present with a wide variety of symptoms, and musculoskeletal involvement is present in $60 \%$ of patients. ${ }^{21}$ In the case described, diagnostic delay was only two days, but that was because of a further request with "appropriate" symptomology. It was felt that this had not been detrimental to the patient.

In the context of demonstrated renal involvement any delay is of concern. It has been shown that in the necrotising vasculitides the most important factor in determining outcome is the presence of renal involvement. ${ }^{22}$ The potential cost to the patient and to the service of a missed diagnosis of glomerulonephritis could be extensive, resulting for example in plasma exchange or dialysis. Locally, a course of seven exchanges would cost approximately $£ 2500$. Furthermore, mortality is increased in patients who present late. Potentially, the savings made by rejecting the $25 \%$ of samples dictated by the gating policy could be outweighed by the cost of a missed diagnosis in a single patient. One further question that remains unanswered and unanswerable by this study is how many patients with ANCA associated vasculitis remained untested and undiagnosed? One would hope the answer was extremely small, because clinical suspicion should drive further investigation, as Sinclair et al have been careful to point out.

The counter argument to the above concern is that the detection of ANCA is only one datum point in the diagnosis. It should be remembered that the presence of an autoantibody is neither essential (not currently included in disease definitions) nor sufficient to make a diagnosis of necrotising vasculitis. ${ }^{16}$ This being the case, it is the responsibility of the clinician to interpret any given pathology test result, not in isolation, but in the context of the patient's case history and other investigations.

\section{SO WHAT APPEAR TO HAVE BEEN THE TRUE EFFECTS OF THE GATING POLICY?}

First, it would appear to have acted as a brake on workload increases. In comparing the workload of Sinclair et al with our own regional reference laboratory in 
Bristol, corrected for differences in the population served, they would appear to perform far fewer tests (perhaps as few as one third) for each head of the population.

Second, it is quite difficult to determine whether the gating policy per se has affected workload balance. We have no data on the workload balance in Sinclair's department before the introduction of the gating policy. In attempting to contrast the gating policy with "open door" centres we have further divergent data. The audit of Edgar et al showed a very high proportion of requests from patients with disorders other than necrotising vasculitis $(73 \%)$ in a clinician led environment. ${ }^{17}$ In contrast, Mandl et al undertook a retrospective study to consider the possible outcome had they applied test ordering guidelines. ${ }^{18}$ These were similar to those used by Sinclair et al. ${ }^{15}$ Mandl et al concluded that using their guidelines would have reduced test ordering by $23 \%$, remarkably similar to the $25 \%$ of Sinclair et al. Mandl and colleagues also suggested that this would improve the PPV and hence diagnostic usefulness. Unfortunately, PPV data were not available in the Sinclair study.

The third unquantifiable outcome is that the gating policy may have led to a more educated clinician base with a clear understanding of the limitations of ANCA testing, leading to self limitation of use.

It would seem then that workload has been reduced but it is still unclear as to whether this has resulted in more appropriate testing. We are left with the impression that a gating policy may work well to control workload, but the effect on clinical usefulness remains speculative. This can only be resolved by a prospective study based on a clear audit of current workload, case mix, and predictive values in a centre using an open door strategy, followed by the introduction of a Sinclair-like rules based policy and subsequent re-audit. Any volunteers?

\section{ACKNOWLEDGEMENTS}

My thanks to P Virgo for his helpful suggestions during the preparation of this manuscript.

J Clin Pathol 2004:57:121-122.

doi: 10.1136/icp.2003.11122

Correspondence to: Mr R J Lock, North Bristol NHS Trust, Southmead Hospital, Bristol BS10 5NB, UK; boblock75@hotmail.com

\section{REFERENCES}

1 The role of the private sector in the NHS Paragraph 141. http://www.parliament.thestationary-office.co.uk/pa/cm200102/cmselect/ cmhealth/308/30802.htm.

2 Van der Woude FJ, Rasmussen N, Lobatto S, et al. Autoantibodies against neutrophils and monocytes: tool for diagnosis and marker of disease activity in Wegener's granulomatosis. Lancet 1985; 1:425-9.

3 Juby C, Johnston C, Davis $\mathrm{P}$, et al. Antinuclear and antineutrophil cytoplasmic antibodies (ANCA) in the sera of patients with Felty's syndrome. Br J Rheumatol 1992;31:185-8.

4 Molnar K, Kovacs L, Kiss M, et al. Antineutrophil cytoplasmic antibodies in patients with systemic lupus erythematosus. Clin Exp Dermatol 2002;27:59-61

5 Targan SR, Landers C, Vidrich A, et al. High-titer antineutrophil cyłoplasmic antibodies in type-1 autoimmune hepatitis. Gastroenterology 1995; 108:1 159-66.

6 Dolman KM, Gans RO, Vervaat TJ, et al. Vasculitis and antineutrophil cytoplasmic autoantibodies associated with propylthiouracil therapy. Lancet 1993;342:651-2.

7 Flores-Suarez LF, Cabiedes J, Villa AR, et al. Prevalence of antineutrophil cytoplasmic autoantibodies in patients with tuberculosis. Rheumatology (Oxford) 2003;42:223-9.

8 Yahya TM, Benedict S, Shalabi A, et al. Antineutrophil cytoplasmic antibody (ANCA) in malaria is directed against cathepsin G. Clin Exp Immunol 1997;110:41-4.
9 Savige J, Davies D, Falk RJ, et al. Antineutrophil cytoplasmic antibodies and associated diseases. A review of the clinical and laboratory features. Kidney Int 2000:57:846-2.

10 Papadakis KA, Targan SR. Serologic testing in inflammatory bowel disease: its value in indeterminate colitis. Current Gastroenterology Reports 1999; 1:482-5.

11 Dubinsky MC, Ofman JJ, Urman M, et al. Clinical utility of serodiagnostic testing in suspected pediatric inflammatory bowel disease. Am J Gastroenterol 2001;96:758-65.

12 Koutroubakis IE, Petinaki E, Mouzas IA, et al. Anti-Saccaromyces cerevisiae mannan antibodies and antineutrophil cytoplasmic autoantibodies in Greek patients with inflammatory bowel disease. Am J Gastroenterol 2001;96:449-54.

13 Roozendaal C, Pogany K, Horst G, et al. Does analysis of the antigenic specificities of antineutrophil cytoplasmic antibodies contribute to their clinical significance in the inflammatory bowel diseases? Scand J Gastroenterol 1999;34:1123-31.

14 Savige J, Gillis D, Benson E, et al. International consensus statement on testing and reporting of antineutrophil cytoplasmic antibodies (ANCA). Am J Clin Pathol 1999:111:507-13.

15 Sinclair D, Saas M, Stevens J. The effect of a symptom related "gating policy" on ANCA requests in routine clinical practice. J Clin Pathol 2004;57:131-4.

16 Davenport A, Lock RJ, Wallington TB, et al. Clinical significance of anti-neutrophil cytoplasm antibodies detected by a standardized indirect immunofluorescence assay. QJ Med 1994:87:291-9.

17 Edgar JDM, McMillan SA, Bruce IN, et al. An audit of ANCA in routine clinical practice. Postgrad Med J 1995;71:605-12.

18 Mandl LA, Solomon DH, Smith EL, et al. Using antineutrophil cytoplasmic antibody testing to diagnose vasculitis: can test-ordering guidelines improve diagnostic accuracy? Arch Intern Med 2002; 162:1509-14.

19 McLaren JS, Stimson RH, McRorie ER, et al. The diagnostic value of anti-neutrophil cytoplasmic antibody testing in a routine setting. QJ Med 2001;94:615-21.

20 Jennette JC, Wilkman AS, Falk RJ. Diagnostic predictive value of ANCA serology. Kidney Int 1998;53:796-8

21 Jennette JC, Falk RJ. Small vessel vasculitis N Engl J Med 1997;337:1512-23.

22 Hogan S, Nachman PH, Wilkman AS, and the Glomerular Diseases Collaborative Network et al. Prognostic markers in patients with antineutrophil cytoplasmic autoantibodyassociated microscopic polyangiitis and glomerulonephritis. J Am Soc Nephrol 1996;7:23-32. 